\title{
Cosmeceutical product consisting of biomimetic peptides: antiaging effects in vivo and in vitro
}

This article was published in the following Dove Press journal:

Clinical, Cosmetic and Investigational Dermatology

7 January 2017

Number of times this article has been viewed

\section{Zarema I Gazitaeva' \\ Anna O Drobintseva ${ }^{2}$ \\ Yongji Chung ${ }^{3}$ \\ Victoria O Polyakova ${ }^{2}$ \\ Igor M Kvetnoy ${ }^{2}$}

'Institute of Beauty Fijie, Moscow, 2Department of Pathomorphology, D.O. Ott Research Institute of

Obstetrics, Gynecology and Reproductology, Saint-Petersburg, Russian Federation; ${ }^{3}$ Caregen Co., Ltd. Research Center, Seoul, South Korea

Correspondence: Anna O Drobintseva Department of Pathomorphology, D.O. Ott Research Institute of Obstetrics, Gynecology and Reproductology, Mendeleevskaya Line, 3, 199034 Saint-

Petersburg, Russian Federation

$\mathrm{Tel}+798 \mathrm{I} \mid 853607$

$\mathrm{Fax}+78123282361$

Email anna.drobintseva@gmail.com
Background: Biomimetic peptides are synthetic compounds that are identical to amino acid sequence synthesized by an organism and can interact with growth factor receptors and provide antiaging clinical effects.

Purpose: The purpose of this study was to investigate the effects of biomimetic peptides on the repair processes in the dermis using a model of cell cultures and in vivo.

Patients and methods: Five female volunteers were subjected to the injection of biomimetic peptides 1 month prior to the abdominoplasty procedure. Cell culture, immunocytochemistry, and confocal microscopy methods were used in this study.

Results: Biomimetic peptides regulate the synthesis of proteins Ki-67, type I procollagen, AP-1, and SIRT6 in cell cultures of human fibroblasts. They contribute to the activation of regeneration processes and initiation of mechanisms that prevent aging. Intradermal administration of complex of biomimetic peptides produces a more dense arrangement of collagen fibers in the dermis and increased size of the fibers after 2 weeks. The complex of biomimetic peptides was effective in the in vivo experiments, where an increase in the proliferative and synthetic activities of fibroblasts was observed.

Conclusion: This investigation showed that the studied peptides have biological effects, testifying the stimulation of reparative processes in the skin under their control.

Keywords: biomimetic peptides, skin aging, collagen, reparation processes, mesotherapy

\section{Introduction}

Skin aging is a complex process that affects all its layers and structure and changes the functional properties of the intracellular matrix.

A wide range of biomimetic peptides with different mechanisms of action can be applied to solve this problem. By imitating the action of naturally occurring growth factors and cytokines, they are able to bind to the specific receptors, regulate gene transcription, and provide a stimulating effect on keratinocytes and fibroblasts. However, the target genes of different peptides are different; hence, the maximum effect is achieved only by the combined application of several biomimetic peptides. ${ }^{1}$

Caregen Co., Ltd. (Anyang-si, Gyeonggi-Do, South Korea) has developed biomimetic peptides based on extensive studies of growth factors since 2002. The term biomimetic peptide as used herein relates to a synthetic agonist of naturally occurring growth factors and completely mimics the action of the parental molecules. The biomimetic peptides are oligopeptides consisting of ten to 15 amino acids and can provide clinical benefits similar to recombinant growth factors, reduce costs, and have greater 
chemical stability. In the production system uses a unique mass production of growth factors provided by transformed bacteria (Escherichia coli system), containing synthetic human genes. Caregen's double-layered encapsulation technology improves penetration of active ingredients into skin and protects the molecules from endogenous proteases, which provides higher efficacy. ${ }^{2}$

Biomimetic peptides have a wide range of applications such as increasing the efficacies of any cosmetic and meso-formulations, including dermal fillers, in antiaging, antipigmentation, hair growth, body fat reduction, and many inflammation-related products, which are also applicable for pharmaceutical purposes. ${ }^{3,4}$ In addition, in the recent decade, an interest in synthetic oligopeptides and their use in diagnosis and treatment of different pathologies has increased dramatically; for example, investigation devoted to influence of short peptides on endothelial growth ${ }^{5}$ or protection of kidney during the cisplatin-induced acute renal failure. ${ }^{6}$

In this study, we identified several molecules associated with aging and skin regeneration, such as Ki-67, type I procollagen, SIRT6, and AP-1. In aging, the number, quality, and quantity of collagen fibers are modified; they become thin, lose their clear organization, and are resorbed. Matrix metalloproteinases (MMP-1, MMP-2, and MMP-3) are key signaling molecules of collagen expression. Many studies have shown that their activity is regulated by AP-1. ${ }^{7-9}$ In parallel, with the degradation of the existing collagen, the activation of factor AP-1 is accompanied with a decrease in procollagen types I and III. AP-1 regulates a number of cellular processes, including differentiation, proliferation, and apoptosis. ${ }^{10}$

There is a decrease in the proliferative activity of fibroblasts during aging. Using the cultivation method, it was shown that fibroblasts of young donors are characterized by twice as many mitoses, whereby one cell (there are $60 \%$ of such type of cells) is able to form a colony of 256 fibroblasts, while in the case of older donors, only $2 \%$ of cells form colonies of similar volume. ${ }^{11}$ The Hayflick phenomenon can be a substantiation of fewer cell divisions, whereby somatic cells without telomerase expression are capable, on average, of only 50 doublings of population, but fibroblasts of older donors have already passed a number of cell cycles prior to isolation in vitro. ${ }^{12}$ There is a correlation between donor age and the replicative lifespan of human cells in culture. ${ }^{13}$ For accuracy, it is better to investigate one serially cultured sample at different periods of time than to examine several different cultured samples. ${ }^{14}$
The protein $\mathrm{Ki}-67$ is present in all active phases of the cell cycle (G1, S, G2, and mitosis), and it is a recognized marker for determining the growth of the cell population.

SIRT6 is a recently identified factor associated with skin aging. DNA repair and control of proliferation are its main functions in the skin, and this protein also participates in inflammation processes. Thus, hyperexpression of SIRT6 leads to increase in TNF- $\alpha$ synthesis through posttranscriptional mechanisms. ${ }^{15}$ SIRT6 modulates telomeric chromatin by Lys-9 deacetylation of histone $\mathrm{H} 3$ and thus prevents telomeric dysfunction and early cellular aging. ${ }^{16}$

The aim of this study was to investigate the influence of biomimetic peptides on the reparation processes in the dermis by the cell culture model in vitro and in vivo.

\section{Patients and methods Human study design}

In the human study, we investigated the effect of revofil aquashine (AQ) that possesses all physical, structural, and biocompatibility properties that are required of a superior gel for use in skin restoring and has a encumbrance certificate. During the clinical study done for encumbrance certification, no major side effects requiring medical attention were observed. AQ contains biomimetic peptides (CG-CGC2, CG-CGC3, Rejuline, Boostrin), free hyaluronic acid, amino acids, multivitamins, and minerals.

AQ was administered to the patients ( $n=5$, age: $45-48$ years) 1 month prior to the abdominoplasty by means of intradermal microinjections into skin of the right half of anterior abdominal wall below the navel (in areas of localized fat deposits that are to be subsequently removed). The samples were received from Caregen Research Center (Seoul, South Korea). As a control, pure (not containing biomimetic peptides) hyaluronic acid was injected simultaneously into a symmetric area to the left.

The treatment and study details were fully explained to the subjects, all of whom signed written informed consent forms pertaining to treatment and participation in this study, which included consent to injection of cosmeceutical product and skin biopsy.

The composition of AQ was supplied in glass syringes with Luer lock. The contents were sterilized using membrane filtration and steam sterilization. A total of $2 \mathrm{~mL}$ of AQ was expected to hold a single procedure; this volume was recommended by Caregen. Needles of $32 \mathrm{G}$ (diameter) were used in the procedure. AQ was injected to a depth of 4-6 mm (depending on skin thickness) and at an angle of $45^{\circ}-90^{\circ}$ to the surface of the skin. 
During the abdominoplasty, skin samples were taken from the injected areas of the abdomen and fixed in $10 \%$ formalin solution. For histological study, specimens were stained with standard hematoxylin and eosin stain. The study was conducted with Caregen Research Ethics Committee approval and patients provided written informed consent.

\section{Cell cultivation}

In the cell culture model, we investigated complex of biomimetic peptides that were part of fillers revofil $A Q$ and $A Q$ brightening (BR). The complex of biomimetic peptides was provided by the supplier in ampoules and was clear, colorless aqueous sterile solution.

The subcultivation of normal human diploid fibroblasts as a model of aging was used in this study in vitro. Human fibroblasts were isolated from the skin of the eyelids, obtained after blepharoplasty. The biopsy was taken from a middle-aged donor (42 years); when the culture reach 7 passage, we classified it as a mature cells, meaning that all processes as energy exchange, cell growth, biosynthesis are in a highest stage, and passage 15 as a senescent culture, thus all the processes mentioned above regress. The senescent cell line was proved by staining for $\beta$-galactosidase (anti- $\beta$-galactosidase antibody; Abcam, Cambridge, UK) as a technique to label senescent cells. Isolation of fibroblasts with enzymatic digestion was carried out using dispase II (Thermo Fisher Scientific, Waltham, MA, USA) at the concentration of $2.4 \mathrm{IU} / \mathrm{mL}$ for 18 hours at $4^{\circ} \mathrm{C}$, and then the epidermis was mechanically separated from the dermis. To obtain a suspension of fibroblasts, the dermis was minced with scissors into pieces of 3-4 $\mathrm{mm}$ and placed in a solution of type I collagenase (Thermo Fisher Scientific) in minimum essential medium (BioloT, Saint-Petersburg, Russia) for 30 minutes at $37^{\circ} \mathrm{C}$. They were seeded at $5 \times 10^{4} \mathrm{cell} / \mathrm{s} /$ well in 12 -well plates and cultured. The obtained culture was tested to specific molecular fibroblast marker vimentin (antivimentin monoclonal antibody; Dako Denmark A/S, Glostrup, Denmark), which confirmed that the investigated culture was skin fibroblasts.

We studied three groups: 1) control group (physiological solution); 2) AQ group with addition of biomimetic peptide components of AQ (acetyl decapeptide-3 [Rejuline], oligopeptide-24 [CG-EGP3], oligopeptide-34 [CG-TGP2], oligopeptide-72 [Boostrin]) in concentration of $2 \mu \mathrm{g} / \mathrm{mL}$; and 3) AQ BR group with addition of biomimetic peptide components of AQ BR (oligopeptide-23 [Flatin], oligopeptide-34 [CG-TGP2], oligopeptide-51 [Purilux], oligopeptide-72 [Boostrin]) in concentration of $2 \mu \mathrm{g} / \mathrm{mL}$.

At passages 7 and 15, cells were seeded on coverslips (15 mm, Thermo Fisher Scientific) fixed with paraformaldehyde
(Sigma-Aldrich Co., St Louis, MO, USA) and incubated with antibodies. The confocal microscope Olympus FV1000 (Olympus Corporation, Tokyo, Japan) was used for visualization of the nuclear markers Ki-67 and SIRT6 in fibroblast culture samples by applying secondary antibodies conjugated with a far red-emitting dye (Alexa Fluor 647; Abcam).

\section{Immunohistochemical study}

In both studies (in vivo and in vitro), we used primary monoclonal antibodies to markers Ki-67 (1:75; Dako Denmark A/S), AP-1 (1:200; Sigma-Aldrich Co.), type I procollagen (1:100, LifeSpan Biosciences, Seattle, WA, USA), and SIRT6 (1:200, Alexa Fluor 647) and biotinylated anti-mouse immunoglobulins (Dako Denmark A/S) as secondary antibodies to provide the immunohistochemical reaction. Visualization of the reaction was carried out using the EnVision Detection System kit (Dako Denmark A/S).

In both cases, morphometric investigation was carried out by using a system of computer analysis of microscopic images, which includes a microscope Olympus BX46, a digital camera (Olympus Corporation), and a personal computer based on Intel Pentium 5 and software Morphology 5.2 (Videotest, Saint-Petersburg, Russia).

We measured the optical density (in cu) and the area of immunopositive expression, which was calculated as the ratio of the area occupied by immunopositive cells (or nuclei) and the total area of cells (or nuclei) in the field of view (in \%).

\section{Statistical analysis}

Statistical analysis of experimental data included calculation of the arithmetic average, the standard deviation, and the confidence interval for each sample, which was performed using the program Statistica 8.0. (Dell; Statistica, Round Rock, TX, USA).

To analyze the distribution of species, we used the Shapiro-Wilk test (Shapiro-Wilk $W$-test). Nonparametric procedures, including one-way analysis of variance (Kruskal-Wallis), were used to verify the statistical homogeneity of several samples. In cases where the variance analysis revealed a statistically significant heterogeneity of multiple samples for subsequent detection of heterogeneous groups (through their pairwise comparisons), we used multiple comparison procedures such as the Mann-Whitney $U$ test.

\section{Results and discussion}

It was shown that in a control sample, with the saline injected, the size of the matrix prevailed in relation to the area of collagen and elastic fibers (Figure 1A). A moderate increase in 
A

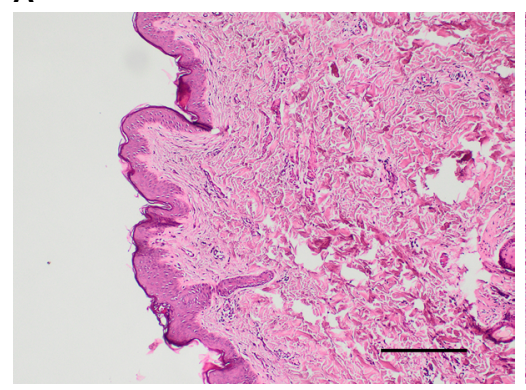

B

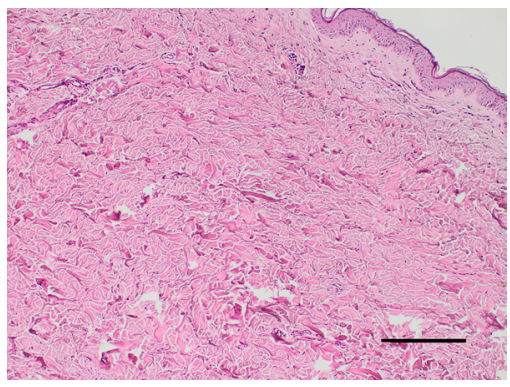

C

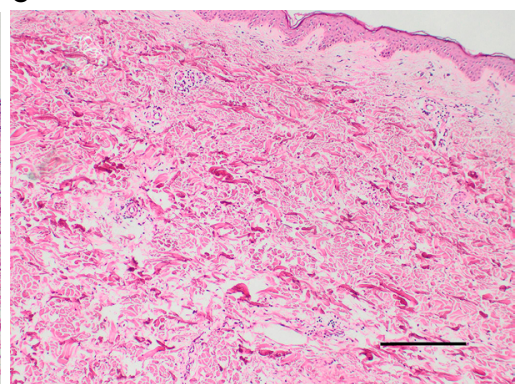

Figure I A histological examination of the skin biopsy specimens, stained with H\&E (magnification $\times 100$ ).

Notes: (A) Control group. (B) Administration of hyaluronic acid. (C) Administration of AQ preparation. Scale bars $200 \mu \mathrm{m}$. Abbreviations: $\mathrm{H} \& \mathrm{E}$, hematoxylin and eosin; $\mathrm{AQ}$, aquashine.
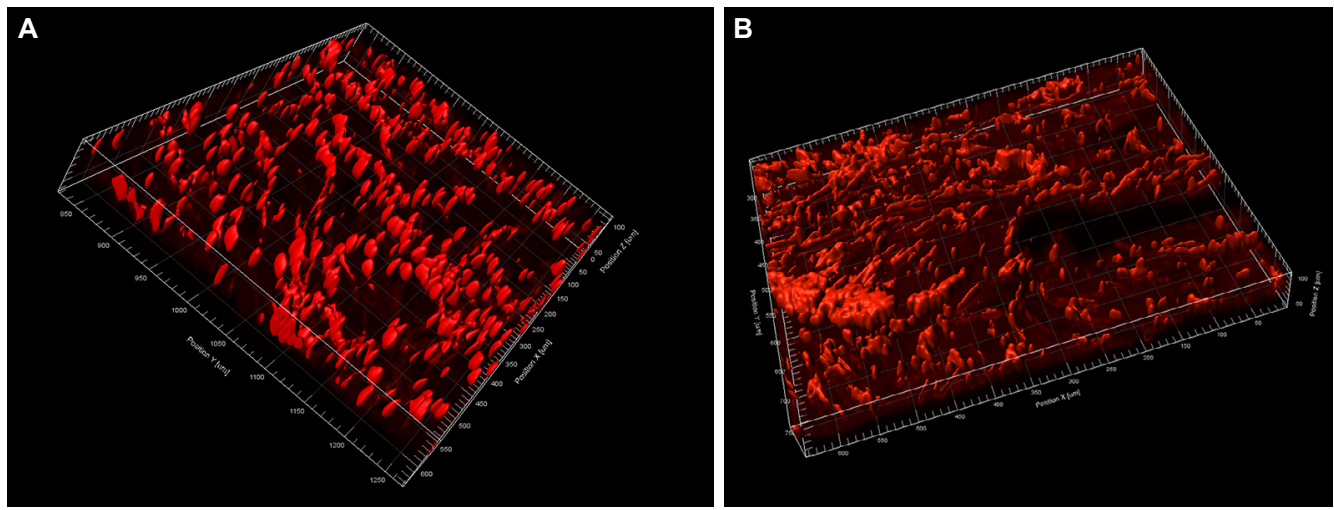

Figure 2 Volumetric reconstruction of marker expression of procollagen (CLSM).

Notes: (A) Control sample. (B) AQ administration.

Abbreviations: CLSM, confocal laser scanning microscopy; $A Q$, aquashine.

the matrix was revealed without any change in the structure of the fibers after administration of hyaluronic acid (Figure 1B). During the histological study, it was revealed that the maximum area of the fibers relative to the matrix was observed in the samples after administration of AQ (Figure 1C) that was determined by the increase in the thickness and density of collagen fibers.

The study of the extracellular matrix component expression of the type I collagen precursor - type I procollagen - was conducted to explore the synthetic activity of dermal fibroblasts. The optical density of expression was measured, as it is the most informative for this marker and characterizes the intensity of immunohistochemical reaction in the samples. Indirectly, it gives an indication of the amount of test substance in the samples.

The maximum value of optical density of the type I procollagen expression was observed in the sample with double injection of $\mathrm{AQ}$, which was $0.285 \mathrm{cu}$, it was also high in the other three samples with a single administration $(0.260 \mathrm{cu}, 0.273 \mathrm{cu}$, and $0.278 \mathrm{cu}$, respectively), indicating a dose-dependent stimulation of the procollagen synthesis and a noticeable effect with an increase in the number of treatment sessions. The minimum value of optical density was observed in the control group and in the samples treated with hyaluronic acid $(0.175 \mathrm{cu}$ and $0.194 \mathrm{cu})$.

The obtained data were confirmed using the confocal laser scanning microscopy studies. The three-dimensional reconstruction of collagen fibers was formed by means of thick slices of the specimens and fluorescent labels conjugated with antibodies to type I procollagen.

By comparing the volume ratios, it was found that the area of newly synthesized type I procollagen with the injection of AQ is two times larger than that in the comparison groups (Figure 2).

The formation and maintenance of collagen fibers not only promotes the synthesis of procollagen by skin fibroblasts but also slows down the process of collagen degradation. The transcription factor AP-1 is one of the main factors regulating the destruction of collagen fibers.

In terms of the area of the transcription factor AP-1 expression, there was a tendency to reduce it in the samples with the AQ administration, where it ranged between $2 \%$ and $6 \%$, while in the samples with the introduction of hyaluronic acid and in the control group, it was within 7\%-14\%. 
We studied the protein SIRT6 to evaluate the activity of skin protective mechanisms from premature aging. It is known that this protein is involved in many processes in the cell, such as DNA repair, telomere elongation, and glycolysis. The area of immunopositive nuclei was assessed by marker for SIRT6 in relation to the total number of nuclei in the dermis in the field of view and expressed as percentage.

The analysis of the data by the area of immunopositive nuclei expression to SIRT6 revealed that there was an increase in the number of immunopositive nuclei compared with that in the control samples even after a single injection of AQ.

Thus, AQ could improve skin aging by affecting bioactive molecules such as SIRT6 and AP-1, promoting division of fibroblasts and expression of collagen de novo. The skin on a skin is rejuvenation through reducing wrinkles and improving skin elasticity by generating new skin cells. Pure hyaluronic acid moisturizes skin and tightens pores through hydration.

Comparative analysis of the injectable preparation effect on the synthetic activity of fibroblasts of mature and senescent skin models showed a significant increase in the area of type I procollagen expression in groups with the administration of $A Q$ and $A Q B R$ compared to the control group (Table 1). The introduction of the investigated preparations promotes the synthesis of collagen by skin fibroblasts de novo. However, the response of fibroblasts was weaker at passage 15 than in younger passages.

These findings were confirmed by experiments in vivo, when the synthesis of collagen in the ultraviolet-protected skin of old people ( 80 years) was reduced by $\sim 75 \%$ in relation to collagen synthesis of young people (18-29 years). ${ }^{17}$

Table I The influence of biomimetic peptides (AQ and AQ BR) on the area of signaling molecules expression in cell culture of fibroblasts

\begin{tabular}{|c|c|c|c|}
\hline \multirow[t]{2}{*}{ Marker peptide } & \multirow[t]{2}{*}{ Group } & \multicolumn{2}{|c|}{ Area of expression (\%) } \\
\hline & & $\begin{array}{l}\text { Passage } 7 \\
\text { (mean } \pm \text { SD) }\end{array}$ & $\begin{array}{l}\text { Passage I5 } \\
\text { (mean } \pm \text { SD) }\end{array}$ \\
\hline \multirow[t]{3}{*}{ Ki-67 } & Control & $20.85 \pm 4$ & $15.63 \pm 3$ \\
\hline & $\mathrm{AQ}$ & $38.47 \pm 5^{*}$ & $31.25 \pm 5^{*}$ \\
\hline & AQ BR & $47.58 \pm 5 *$ & $33.30 \pm 5 *$ \\
\hline \multirow[t]{3}{*}{ SIRT6 } & Control & $9.65 \pm 0,5$ & $2.05 \pm 0.4$ \\
\hline & $\mathrm{AQ}$ & $\mathrm{II} .36 \pm \mathrm{I}^{*}$ & $9.29 \pm 1 *$ \\
\hline & $\mathrm{AQ} B R$ & $12.13 \pm 2 *$ & $10.08 \pm 1 *$ \\
\hline \multirow[t]{3}{*}{ Type I procollagen } & Control & $17.58 \pm 2$ & $3.37 \pm 1$ \\
\hline & $\mathrm{AQ}$ & $22.57 \pm 2 *$ & $12.07 \pm 1 *$ \\
\hline & $\mathrm{AQ} B R$ & $24.82 \pm 2 *$ & $10.63 \pm 2 *$ \\
\hline \multirow[t]{3}{*}{ AP-I } & Control & $5.12 \pm 0.5$ & $7.12 \pm 0.5$ \\
\hline & $\mathrm{AQ}$ & $2.34 \pm 0.5^{*}$ & $4.98 \pm 1 *$ \\
\hline & $\mathrm{AQ} B R$ & $2.08 \pm 0.1 *$ & $6.18 \pm 1$ \\
\hline
\end{tabular}

Note: *Comparison versus control, $P<0.05$.

Abbreviations: $A Q$, aquashine, $S D$, standard deviation; $B R$, brightening.

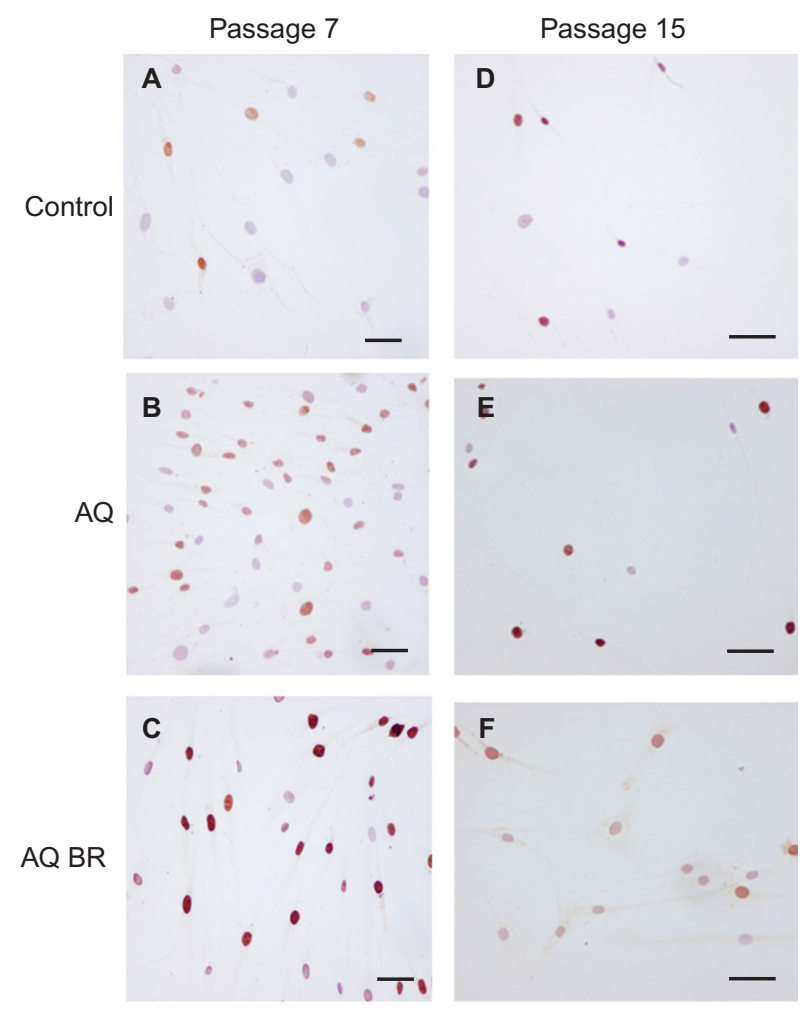

Figure 3 Immunohistochemical EnVision staining of fibroblast cell culture. Notes: Immunoreactivity for $\mathrm{Ki}-67$ was observed in mature cell culture (passage 7) in the control group (A), in group with addition of biomimetic peptides from $A Q(B)$, and in group with addition of biomimetic peptides from AQ BR (C). Immunopositive nucleus was revealed in a senescent cell culture (passage 15) in the control group (D), in the AQ group (E), and in the AQ BR group (F). Scale bars: $100 \mu \mathrm{m}$. Magnification $=200 \times$.

Abbreviations: $A Q$, aquashine; $B R$, brightening.

Stimulating effects of AQ and AQ BR cosmeceutical products on the proliferative activity of human skin fibroblasts were detected in cell culture. Even in aging cell cultures, the parameter of the relative number of immunopositive nuclei to Ki-67-marker was two times higher than that in the control group (Figure 3).

The study of the transcription factor AP-1 in the cell culture of the skin fibroblasts revealed a statistically significant decrease in the area of expression in groups with the introduction of AQ and AQ BR by 2.1 and 3.1 times, respectively, than that in the control group. Statistically significant differences were identified only at the effect of $\mathrm{AQ}$, indicating dermatogenic protective action of the investigated substances, realized through antidegradation of collagen fibers.

The expression of SIRT6 protein was revealed by cell cultivation of skin fibroblasts in all the three groups. The maximum amount of immunopositive nuclei was observed in the culture of fibroblasts incubated with AQ BR at passage 7 , but this value was not statistically different from the data of the group with the introduction of AQ. 
Biomimetic peptides contributed to an increase in the area of immunopositive nuclei by $79 \%$ after incubation with AQ BR and by $77 \%$ with the introduction of AQ (Table 1 ). This indicates the start of the mechanisms of DNA stability maintenance, protection of telomeres, and other processes preventing aging.

In the future investigation, it will be interesting to use real-time polymerase chain reaction and enzyme-linked immunosorbent assay methods to confirm data obtained in this study and expand knowledge of action of biomimetic peptides on skin aging processes.

\section{Conclusion}

The immunohistochemical studies revealed the molecular mechanisms of the clinical effects (lifting effect, remodeling of the dermis with filling of small wrinkles, dermoprotective effect on the elastic matrix of the skin, improvement in skin resistance to aggressive environmental factors and mutations) observed with intradermal administration of AQ both in vitro (in cell culture of human fibroblasts) and in vivo (in biopsies of human skin).

\section{Disclosure}

The authors report no conflicts of interest in this work.

\section{References}

1. Penning A [webpage on the Internet]. Biomimetics: beauty ingredients that mimic bio functions. GCI Magazine. 2013 Oct 14; 3. Available from: http://www.gcimagazine.com/business/rd/ingredients/Biomimetics-Beauty-Ingredients-That-Mimic-Bio-Functions-230110461.html. Accessed August 23, 2015.

2. Caregen Co., LTD. [homepage on the Internet]. Growth Factors and Biomimetic Peptides. Seul: Caregen-Dynamic, Progressive and R\&DBased Company; 2015. Available from: http://www.caregen.co.kr/. Accessed September 11, 2015.
3. Oh SJ, Kim K, Lim CJ. Protective properties of ginsenoside Rb1 against UV-B radiation-induced oxidative stress in human dermal keratinocytes. Pharmazie. 2015;70(6):381-387.

4. Grosicki M, Latacz G, Szopa A, Cukier A, Kieć-Kononowicz K. The study of cellular cytotoxicity of argireline - an anti-aging peptide. Acta Biochim Pol. 2014;61(1):29-32.

5. Malinin VV, Durnova AO, Polyakova VO, Kvetnoi IM. Effects of LysGlu-Trp peptide on cell-cell interactions and vascular endothelium proliferation under normal conditions and during atherosclerosis. Bull Exp Biol Med. 2014;157(3):324-326.

6. Zamorskii II, Shchudrova TS, Lin'kova NS, Nichik TE, Khavinson VKH. Peptides restore functional state of the kidneys during cisplatin-induced acute renal failure. Bull Exp Biol Med. 2015;159(6):736-739.

7. Bickers DR, Athar M. Oxidative stress in the pathogenesis of skin disease. J Investigative Dermatol. 2006;126(12):2565-2575.

8. Helfrich YR, Sachs DL, Voorhees JJ. Overview of skin aging and photoaging. Dermatol Nurs. 2008;20(3):177-183; quiz 184.

9. Chen YJ, Chang LS. Simvastatin induces NFאB/p65 down-regulation and JNK1/c-Jun/ATF-2 activation, leading to matrix metalloproteinase-9 (MMP-9) but not MMP-2 down-regulation in human leukemia cells. Biochem Pharmacol. 2014;92(4):530-543.

10. Ameyar M, Wisniewska M, Weitzman JB. A role for AP-1 in apoptosis: the case for and against. Biochem. 2003;85(8):747-752.

11. Covas DT, Panepucci RA, Fontes AM, et al. Multipotent mesenchymal stromal cells obtained from diverse human tissues share functional properties and gene expression profile with CD146+ perivascular cells and fibroblasts. Exp Hematol. 2008;36(5):642-654.

12. Grytsenko MA. Fibroblasts in the course of development and aging of an organism. J V.N.Karazin Kharkiv Nat Univ Ser Biol. 2013; 17(1056):10-16.

13. Cristofalo VJ, Allen RG, Pignolo RJ, Martin BG, Beck JC. Relationship between donor age and the replicative lifespan of human cells in culture: a reevaluation. Proc Natl Acad Sci USA. 1998;95(18):10614-10619.

14. Phipps SM, Berletch JB, Andrews LG, Tollefsbol TO. Aging cell culture. Meth Observ. 2007;371:9-19.

15. Seravallo M, Jagdeo J, Glick SA, Siegel DM, Brody NI. Sirtuins in dermatology: applications for future research and therapeutics. Arch Dermatol Res. 2013;305(4):269-282.

16. Michishita E, McCord RA, Berber E, et al. SIRT6 is a histone H3 lysine 9 deacetylase that modulates telomeric chromatin. Nature. 2008; 452(7186):492-496.

17. Varani J, Dame MK, Rittie L, et al. Decreased collagen production in chronologically aged skin: roles of age-dependent alteration in fibroblast function and defective mechanical stimulation. Am J Pathol. 2006;168(6): 1861-1868.
Clinical, Cosmetic and Investigational Dermatology

\section{Publish your work in this journal}

Clinical, Cosmetic and Investigational Dermatology is an international, peer-reviewed, open access, online journal that focuses on the latest clinical and experimental research in all aspects of skin disease and cosmetic interventions. This journal is included on PubMed. The manuscript management system is completely online

\section{Dovepress}

and includes a very quick and fair peer-review system, which is all easy to use. Visit http://www.dovepress.com/testimonials.php to read real quotes from published authors 